\title{
Comparison of Intraoperative $\gamma$-Probe Imaging and Postoperative SPECT/CT in Detection of Sentinel Nodes Related to the Ovary
}

\author{
Stéphanie C.J.M. Speth ${ }^{1}$, Roy F.P.M. Kruitwagen ${ }^{1,2}$, Marjolein Kleppe ${ }^{1}$, Ivo N.A. Pooters ${ }^{3}$, Toon Van Gorp ${ }^{1,2}$, \\ Brigitte F.M. Slangen ${ }^{1,2}$, and Boudewijn Brans ${ }^{3}$ \\ ${ }^{I}$ Department of Obstetrics and Gynaecology, Maastricht University Medical Centre, Maastricht, The Netherlands; ${ }^{2}$ GROW—School \\ for Oncology and Developmental Biology, Maastricht, The Netherlands; and ${ }^{3}$ Department of Radiology and Nuclear Medicine, \\ Maastricht University Medical Centre, Maastricht, The Netherlands
}

\begin{abstract}
Tracer injection into ovarian ligaments has been shown to detect sentinel nodes (SNs) in patients with ovarian cancer. To determine the possibility that SNs are missed, this feasibility study compared their detection during surgery with their detection on postoperative SPECT/CT. Methods: In 8 patients (with either ovarian or endometrial cancer), after a staging lymphadenectomy including resection of SNs related to the ovary, SPECT/CT was performed within $24 \mathrm{~h}$. Results: SPECT/CT identified hotspots in 4 patients at sites where SNs were resected. In 6 patients, additional sites were found, mainly in the pelvic region. Conclusion: Discrepancies between the $\gamma$-probe and SPECT/CT may be due to missed SNs during surgery, but with respect to pelvic hotspots, in most cases they are more probably related to remnants of tracer at injection sites. With respect to sites where SNs were resected, remaining hotspots may have been caused by residual lymphatic flow after resection.
\end{abstract}

Key Words: sentinel node; ovarian cancer; SPEC/CT

J Nucl Med 2017; 58:243-245

DOI: 10.2967/jnumed.116.183426

$\mathbf{I}_{\mathrm{n}}$ clinical early-stage epithelial ovarian cancer, the International Federation of Obstetrics and Gynaecology recommends a staging procedure that includes a complete pelvic and paraaortic lymphadenectomy (1). However, a complete lymphadenectomy is associated with morbidity, including nerve and vessel injury, increased blood loss, increased operating time, and the formation of lymph cysts and lymphedema (2-4). A sentinel node (SN) procedure may play an important role in the management of epithelial ovarian cancer. Few studies in patients with ovarian cancer have evaluated SNs, partly because of the risk of tumor dissemination associated with the injection of tracers into the ovarian cortex (5-9). In a previous pilot study, we avoided possible tumor cell spillage by injection of tracers into the ovarian ligaments rather than into the ovarian cortex (10). We identified at least one $\mathrm{SN}$ in all patients with suspected ovarian cancer $(n=21)(11)$. However, by the nature of

Received Sep. 1, 2016; revision accepted Sep. 14, 2016.

For correspondence or reprints contact: Roy F.P.M. Kruitwagen, Maastricht University Medical Centre, P.O. Box 5800, 6202 AZ Maastricht, The Netherlands.

E-mail: r.kruitwagen@mumc.nl

Published online Oct. 13, 2016.

COPYRIGHT (C) 2017 by the Society of Nuclear Medicine and Molecular Imaging. this approach without preoperative imaging of the $\mathrm{SN}$ locations, we were conscious that the single use of a $\gamma$-probe or blue color to detect hotspots during surgery could lead to missed SNs. With this in mind, in the present feasibility study we describe patients in whom, after resection of SNs identified by $\gamma$-probe and blue dye, postoperative SPECT/CT was performed.

\section{MATERIALS AND METHODS}

\section{Patients}

As described previously, patients diagnosed with a pelvic mass suggestive of a malignant ovarian tumor, and patients with high-grade endometrial cancer planned for staging laparotomy, were eligible to participate in the study $(10,11)$. We included both patients with suspected ovarian malignancy and patients with a high-grade uterine carcinoma. The latter group could be included because these patients undergo the same surgical procedure: total abdominal hysterectomy with bilateral salpingo-oophorectomy and a pelvic and paraaortic lymphadenectomy or lymph node sampling. All patients provided fully informed consent in writing before enrollment in the study, and the protocol was approved by the Local Ethics Committee (approval NL40323.068.12) (clinical trial registration number NCT01734746).

\section{Surgical Procedure}

The surgical procedure has been described in detail in previous reports $(10,11)$. Briefly, in patients with an adnexal tumor, tracer was injected on the dorsal and ventral sides of both the proper ovarian ligament and the suspensory ligament. Each of the 4 injections consisted of a standard dose of $0.2-0.5 \mathrm{~mL}$ of undiluted blue dye (Patent Blue V, $25 \mathrm{mg} / \mathrm{mL}$; Guerbet Nederland B.V.) and $20 \mathrm{MBq}$ (total dose of $80 \mathrm{MBq}$ ) of ${ }^{99 \mathrm{~m} T c-}$ labeled albumin nanocolloid, of particle size less than $80 \mathrm{~nm}$ (Nanocoll; GE Healthcare). Fifteen minutes later, the adnexal mass was removed and sent for frozen-section analysis. If the ovarian mass was found to be malignant, the retroperitoneal space was opened, SNs were localized with the $\gamma$-probe or visually (blue dye) and resected. After removal of the SNs, a complete standard staging procedure was performed including a comprehensive sampling of other lymph nodes in various anatomic locations.

For the detection of possible SNs related to the ovary, tracer injection in patients with endometrial cancer was identical to that in patients with an ovarian tumor, with injection on the right or left side. Fifteen minutes after injection, a complete hysterectomy with bilateral salpingo-oophorectomy was performed, followed by retroperitoneal exploration to identify and resect the SNs. In addition, standard pelvic and paraaortal lymph node sampling was performed according to protocol. 


\section{Postoperative SPECT/CT}

Depending on the condition and mobility of the patient, a scintigram was performed within $24 \mathrm{~h}$ after surgery. SPECT/CT images were acquired using a standard SPECT/CT camera (Precedence SPECT/6-slice CT scanner; Philips), equipped with dual $1.6-\mathrm{cm} \gamma$-detectors and lowenergy general-purpose collimators. SPECT/CT data of the abdomen and pelvis were obtained by a noncircular orbit, a $64 \times 64 \times 16$ matrix, and 64 angles over $180^{\circ}$ and $30 \mathrm{~s}$ per stop, after which CT scanning was performed $(120 \mathrm{kV}, 30 \mathrm{mAs}, 2-\mathrm{mm}$-thick slices).

\section{RESULTS}

\section{Patients}

In 8 patients ( 5 patients with ovarian cancer [patients 1-5] and 3 patients with endometrial cancer [patients 6-8]) in whom a surgical staging lymphadenectomy including resection of SNs was performed, a SPECT/CT scan was acquired within $24 \mathrm{~h}$ postoperatively. The 5 patients with ovarian cancer were incorporated in a previous report describing the feasibility of the intraoperative $\mathrm{SN}$ detection (11). In none of the patients did a preoperative CT scan, acquired as part of the diagnostic work-up, show signs of tumor spread (either intraabdominal or pathologic lymph nodes). The 3 patients with endometrial cancer showed normal ovaries on CT and during surgery.

\section{SN Detection}

Figure 1 shows the hotspot locations identified with the $\gamma$-probe transperitoneally, with the $\gamma$-probe during retroperitoneal exploration, and with SPECT/CT postoperatively. At least one hotspot was detected in each of these 8 patients using the $\gamma$-probe transperitoneally. During retroperitoneal exploration, 20 hotspots were detected with the $\gamma$-probe. In 7 patients (88\%), SNs corresponded to a transperitoneally identified hotspot, with 5 patients (patients $1,2,5,6$, and $7 ; 71 \%$ ) having more than one $\mathrm{SN}$ in this region. In addition, 2 patients (patients 1 and $3 ; 25 \%$ ) had SNs in regions not initially identified transperitoneally. In 1 patient, the transperitoneally detected hotspot in the low aortocaval region could not be recognized at retroperitoneal exploration. In contrast, in this patient an $\mathrm{SN}$ was detected in the high aortocaval region. In 2 patients (patients 1 and 6; $25 \%$ ), the SNs were also visible by blue dye.

Postoperative SPECT/CT showed at least one hotspot in all 8 patients, for a total of 11 hotspots. In 4 patients, 5 hotspots in total ( 1 each in patients 1,6 , and 7 and 2 in patient $5 ; 45 \%$ ) were identified at sites where SNs were resected ( 3 aortocaval and 2 pelvic). The remaining 6 hotspots were in the pelvic region. Figure 2 shows the lymphatic drainage from a right ovarian tumor after resection of an SN at the aortocaval region, illustrated by SPECT/CT.

\section{Histopathology}

All 20 tissue specimens related to transperitoneally identified hotspots contained at least one lymph node. In one patient, a single tissue specimen contained 2 lymph nodes (Fig. 1). In one patient with endometrial cancer, lymph node metastases were found in both SNs at histopathology (Fig. 1).

\section{DISCUSSION}

In the present feasibility study, in patients with an ovarian or high-grade endometrial cancer, detection of SNs by $\gamma$-probe during surgery by injecting tracers in the ovarian ligaments was compared with detection by postoperative SPECT/CT imaging. In 4 patients, one or more hotspots could still be identified at locations where the SNs were resected. Furthermore, in 6 patients hotspots were detected in pelvic regions that were not identified during surgery.

As previously reported, retroperitoneal exploration was more successful than transperitoneal examination in detecting SNs (11). This finding could be explained by better, more precise accessibility of the lymph node locations but possibly also by a longer interval after injection of the tracer. The inability of SPECT/CT to detect these hotspots was obviously due to the resection of these SNs during surgery. However, in 4 patients, SPECT/CT detected residual activity at these sites. This finding may have been caused by residual lymphatic flow containing tracer after resection, with tracer fluid accumulating at the site of the $\mathrm{SN}$ resection.

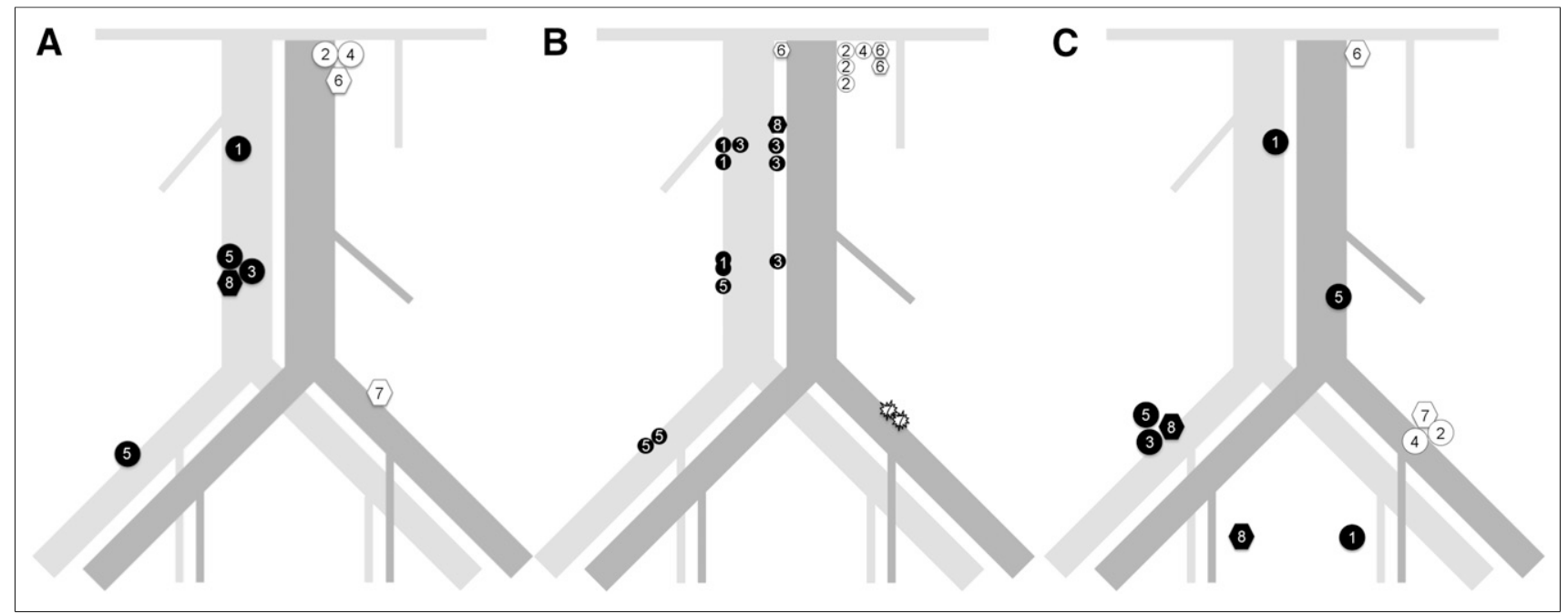

FIGURE 1. Detection of SNs in patients with malignant tumor. (A) Transperitoneal detection of hotspots. (B) Hotspot localizations during retroperitoneal exploration. (C) Localizations of hotspots seen on SPECT/CT postoperatively. Numbers represents study numbers given to patients with malignant tumor. Black figures = patients with injection on right side; white figures = patients with injection on left side; circles $=$ patients with ovarian cancer; hexagons $=$ patients with endometrial cancer; stars $=$ SNs containing metastasis. 

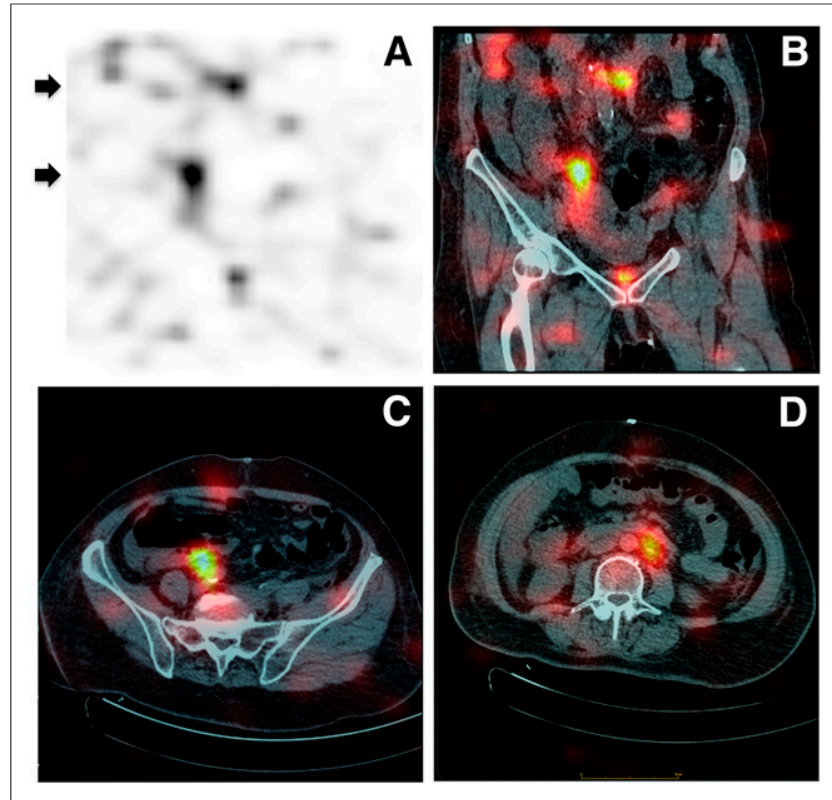

FIGURE 2. Example of lymphatic drainage from right ovarian tumor (patient 5) as illustrated by SPECT/CT. Upper and lower arrows on maximum-intensity projection $(\mathrm{A})$ and coronal slice $(\mathrm{B})$ correspond to respective transversal position, that is, iliaca externa $(C)$ and paraaortal low (D). Paraaortal lymph node in this patient was resected during operation.

The marked discrepancy between the $\gamma$-probe and SPECT/ CT-in that some hotspots in the pelvic region were detected only by SPECT/CT - can most probably be explained by the injection sites of the tracer. These hotspots were located mainly at the level of the external iliac vessels or the psoas muscle, where the suspensory ligament reaches the pelvis and passes close to the iliac vessels. Although hysterectomy and bilateral salpingo-oophorectomy also included resection of the sites of tracer injection in the suspensory ligament near the psoas muscle, the latter could not always be performed completely. Near the pouch of Douglas, 2 hotspots were found that may have been remnants of tracer fluid that leaked during injection. Postoperative lymphatic mapping with SPECT/CT, as in our study, was intended only to validate the $\mathrm{SN}$ procedure. One technique that may be of great interest is the intraoperative use of a portable $\gamma$-camera, which may result in real-time imaging (12). This method may provide an overview of all radioactive hotspots throughout the entire surgical field.

\section{CONCLUSION}

SNs may be safely detected in patients with epithelial ovarian cancer by injecting the tracers into the ovarian ligaments. However, there were discrepancies in SN detection between intraoperative $\gamma$-probe imaging and postoperative SPECT/CT. Whether the hotspots visualized on SPECT/CT were actually missed SNs cannot be determined from these data. Large, multicenter trials are needed to compare the histology of SNs and non-SNs and to evaluate whether intraoperative imaging with a mobile $\gamma$-camera improves the accuracy of SN identification. This should also be related to the significant developments in the use of fluorophores and fluorescent signatures, such as in prostatic cancer, and hybrid tracers.

\section{DISCLOSURE}

No potential conflict of interest relevant to this article was reported.

\section{REFERENCES}

1. Benedet JL, Bender H, Jones H III, Ngan HY, Pecorelli S. FIGO staging classifications and clinical practice guidelines in the management of gynecologic cancers. FIGO Committee on Gynecologic Oncology. Int J Gynaecol Obstet. 2000;70:209-262.

2. Maggioni A, Benedetti Panici P, Dell'Anna T, et al. Randomised study of systematic lymphadenectomy in patients with epithelial ovarian cancer macroscopically confined to the pelvis. Br J Cancer. 2006;95:699-704.

3. Ditto A, Martinelli F, Reato C, et al. Systematic para-aortic and pelvic lymphadenectomy in early stage epithelial ovarian cancer: a prospective study. Ann Surg Oncol. 2012;19:3849-3855.

4. van de Poll-Franse LV, Pijnenborg JM, Boll D, et al. Health related quality of life and symptoms after pelvic lymphadenectomy or radiotherapy vs. no adjuvant regional treatment in early-stage endometrial carcinoma: a large populationbased study. Gynecol Oncol. 2012;127:153-160.

5. Negishi H, Takeda M, Fujimoto T, et al. Lymphatic mapping and sentinel node identification as related to the primary sites of lymph node metastasis in early stage ovarian cancer. Gynecol Oncol. 2004;94:161-166.

6. El-Ghobashy AE, Saidi SA. Sentinel lymph node sampling in gynaecological cancers: techniques and clinical applications. Eur J Surg Oncol. 2009;35:675-685.

7. Nyberg RH, Korkola P, Maenpaa J. Ovarian sentinel node: is it feasible? Int J Gynecol Cancer. 2011;21:568-572.

8. Giammarile F, Vidal-Sicart S, Valdes Olmos RA. Uncommon applications of sentinel lymph node mapping: urogenital cancers. Q J Nucl Med Mol Imaging. 2014;58:161-179.

9. Giammarile F, Bozkurt MF, Cibula D, et al. The EANM clinical and technical guidelines for lymphoscintigraphy and sentinel node localization in gynaecological cancers. Eur J Nucl Med Mol Imaging. 2014;41:1463-1477.

10. Kleppe M, Van Gorp T, Slangen BFM, et al. Sentinel node in ovarian cancer: study protocol for a phase 1 study. Trials. 2013;14:47.

11. Kleppe M, Brans B, Van Gorp T, et al. The detection of sentinel nodes in ovarian cancer: a feasibility study. J Nucl Med. 2014;55:1799-1804.

12. Vermeeren L, Meinhardt W, Bex A, et al. Paraaortic sentinel lymph nodes: toward optimal detection and intraoperative localization using SPECT/CT and intraoperative real-time imaging. J Nucl Med. 2010;51:376-382. 